\title{
Microstructural Abnormalities of Basal Ganglia and Thalamus in Bipolar and Unipolar Disorders: A Diffusion Kurtosis and Perfusion Imaging Study
}

\author{
Lianping Zhao ${ }^{1,2 *}$, Ying Wang ${ }^{1,3 *} \otimes$, Yanbin $\mathrm{Jia}^{4}$, Shuming Zhong ${ }^{4}$, \\ Yao Sun ${ }^{1}$, Zhifeng Zhou', Zhongping Zhang ${ }^{5}$, and Li Huang ${ }^{1}$ \\ ${ }^{1}$ Medical Imaging Center, First Affiliated Hospital of Jinan University, Guangzhou, China \\ ${ }^{2}$ Department of Radiology, Gansu Provincial Hospital, Gansu, China \\ ${ }^{3}$ Clinical Experimental Center, First Affiliated Hospital of Jinan University, Guangzhou, China \\ ${ }^{4}$ Department of Psychiatry, First Affiliated Hospital of Jinan University, Guangzhou, China \\ ${ }^{5}$ General Electric Healthcare, Shanghai, China
}

Objective Bipolar disorder (BD) is often misdiagnosed as unipolar depression (UD), leading to mistreatment and poor clinical outcomes. However, little is known about the similarities and differences in subcorticalgray matter regions between BD and UD.

Methods Thirty-five BD patients, 30 UD patients and 40 healthy controls underwent diffusional kurtosis imaging (DKI) and three dimensional arterial spin labeling (3D ASL). The parameters including mean kurtosis (MK), axial kurtosis (Ka), radial kurtosis (Kr), fractional anisotropy (FA), mean diffusivity (MD), axial diffusivity (Da), radial diffusivity (Dr) and cerebral blood flow (CBF) were measured by using regions-of-interest analysis in the caudate, putamen and thalamus of the subcortical gray matter regions.

Results UD exhibited differences from controls for DKI measures and CBF in the left putamen and caudate. BD showed differences from controls for DKI measures in the left caudate. Additionally, BD showed lower Ka in right putamen, higher MD in right caudate compared with UD. Receiver operating characteristic analysis revealed the $\mathrm{Kr}$ of left caudate had the highest predictive power for distinguishing UD from controls.

Conclusion The two disorders may have overlaps in microstructural abnormality in basal ganglia. The change of caudate may serve as a potential biomarker for UD.

Psychiatry Investig 2017;14(4):471-482

Key Words Bipolar disorder, Depressive disorder, Major, Brain imaging/neuroimaging, Diffusion, Perfusion imaging.

\section{INTRODUCTION}

Bipolar disorder (BD) is characterized by alternating episodes of mania and depression. The depressive episodes of $\mathrm{BD}$ are the most common mood manifestation of the illness. This is dramatically demonstrated in a long-term follow-up

Received: May 29, 2016 Revised: June 23, 2016

Accepted: July 25, 2016 Available online: May 16, 2017

$\square$ Correspondence: Li Huang, MD

Medical Imaging Center, First Affiliated Hospital of Jinan University, Guangzhou 510630, China

Tel: +8620 38688005, Fax: +86 20 85228523, E-mail: cjr.huangli@vip.163.com

$\square$ Correspondence: Ying Wang, PhD

Clinical Experimental Center, First Affiliated Hospital of Jinan University, Guangzhou 510630, China

Tel: +86 20 38688071, Fax: +86 20 85228523, E-mail: johneil@vip.sina.com

*Lianping Zhao and Ying Wang contributed equally to this work.

@ This is an Open Access article distributed under the terms of the Creative Commons Attribution Non-Commercial License (http://creativecommons.org/licenses/by$\mathrm{nc} / 4.0$ ) which permits unrestricted non-commercial use, distribution, and reproduction in any medium, provided the original work is properly cited. of 146 patients with bipolar disorder, which found that nearly half of the time over 13 years patients were symptomatic in some fashion, overwhelmingly with depressive symptoms. ${ }^{1}$ It was reported that nearly $69 \%$ of BD patients had been misdiagnosed, with the most frequent misdiagnosis being major depressive disorder or recurrent unipolar depression (UD), leading to inadequate treatment, huge medical costs and poor clinical outcomes. ${ }^{2}$ UD is characterized only by episodes of depression, and its lifetime prevalence ranges from $10 \%$ to $30 \%{ }^{3,4}$ However, consistent biomarkers distinguishing the two disorders have been elusive. Accumulating evidence suggests a wide spread structural and functional brain abnormalities in patients with BD and UD. ${ }^{5}$ But only a few neuroimaging studies have already been published directly comparing the two disorders.

Basal ganglia and thalamus are potential key brain regions involved in mental disorders. These regions have been related 
to cognitive and emotional processing especially via corticobasal ganglia, cortico-thalamic and cortico-limbic circuits, and critically implicated in executive functions that are commonly impaired in psychotic conditions. ${ }^{6-10}$ These structures have traditionally been viewed as motor processing nuclei such as Parkinson's disease $\mathrm{e}^{8,11,12}$ and Huntington's disease. ${ }^{13,14}$ However, functional neuroimaging evidence has implicated them in more complex cognitive and affective processes that are fundamental for a range of human activities. ${ }^{6,715}$ Recently, it has been suggested that the basal ganglia and thalamus may play an important role in the development of BD and UD. ${ }^{16,17}$ Structural magnetic resonance imaging (MRI) studies have indicated volumes reduction and shape changes in these regions in the two disorders. ${ }^{16,18-22}$ Functional MRI studies have demonstrated functional impairment in these structures in $\mathrm{BD}^{23}$ and UD. ${ }^{24}$ However, because of the limits of conventional MRI technology, far too little attention has been paid to the microstructural changes and perfusional abnormalities in basal ganglia and thalamus which might help to clarify the neuropathological mechanisms of the two disorders.

In recent years, diffusional kurtosis imaging (DKI), a relatively new diffusional technique, has been progressively used for brain studies. Compared with the traditional diffusion tensor imaging (DTI) technique, DKI characterizes nonGaussian water distribution while conventional DTI characterizes Gaussian diffusion in neural tissue. ${ }^{25-27} \mathrm{DKI}$ is a straightforward extension of DTI which only requires minor changes in data acquisition and processing. ${ }^{25,28}$ Based on the same type of pulse sequences as employed for conventional diffusion weighted imaging (DWI), but the required $\mathrm{b}$ values are somewhat larger than those usually employed. Research shows that DKI can provide more information about the microstructural abnormalities of brain tissue than traditional DTI, ${ }^{25,29,30}$ and is not confined to anisotropic environments, therefore, the microstructural integrity of both white and gray matter can be quantitatively analyzed, even in the regions where there are crossing fibers. ${ }^{25}$ This sensitivity to gray matter may be of great importance in the examination of the microstructural integrity in the basal ganglia and thalami since these structures are all gray matters. Based on DKI data, in addition to the conventional diffusion metrics, such as fractional anisotropy (FA) and mean diffusivity (MD), axial diffusivity (Da) and radial diffusivity (Dr), the kurtosis parameters, including mean kurtosis (MK), axial kurtosis (Ka) and radial kurtosis $(\mathrm{Kr})$, were obtained to quantify the deviation of water diffusion from a homogeneous, unrestricted, free distribution. Since DKI first introduced by Jensen et al., ${ }^{28}$ it has shown promising preliminary results for several brain diseases including temporal lobe epilepsy, ${ }^{27}$ Parkinson disease, ${ }^{31}$ attention-deficit hyperactivity disorder, ${ }^{32}$ as well as schizophre- nia. ${ }^{33}$ So far, however, there has been little study related to $\mathrm{BD}$ and UD by using DKI to evaluate the microstructural integrity of the subcorticalgray matter regions.

Some recent studies ${ }^{34-36}$ conducting functional investigations of BD or UD have also reported impairment of perfusion and have demonstrated that changes in cerebral blood flow $(\mathrm{CBF})$ might play a role in the development of the two disorders. Three dimensional arterial spin labeling (3D ASL) is a relatively novel developed MR perfusion technique that can noninvasively assess CBF by using arterial blood water as an endogenous freely diffusible tracer. However, only limited literature uses this technique to investigate the perfusion changes of these disorders. ${ }^{37,38}$ Our previous cross-sectional study ${ }^{36}$ shows preliminary results uses 3D ASL in cerebellum in the two disorders.

In the present study, we aimed to investigate the possible abnormalities of basal ganglia, and thalamusin BD-II and UD by using DKI and 3D ASL. We hypothesized that the two patient groups would share similarities and exhibit differences in microstructural and perfusional changes in these regions. To our best knowledge, this is the first study to show the microstructural integrity in the subcortical gray matter regions in vivo in the two disorders, which might help differentiate $\mathrm{BD}$-II from UD depression.

\section{METHODS}

\section{Subjects}

Thirty-five patients with depressed BD and 30 patients with UD in depressive episode were recruited from the in-patient unit of the psychiatry department, First Affiliated Hospital of Jinan University, Guangzhou, China. The patients were aged from 18 to 55 years. All participants met DSM-IV criteria based on the Structured Clinical Interview for DSM-IV Patient Edition (SCID-P) for BD and UD with a score 18 or greater on the 17-item Hamilton Depression Rating Scale (HDRS). Patients with other Axis-I psychiatric disorders, a history of organic brain disorder, neurological disorders, mental retardation, cardiovascular diseases, alcohol/substance abuse, pregnancy, or any physical illness were excluded from the study. None of the patients had ever received electroconvulsive therapy prior to participating in the study. At the time of the study, 20 patients in the BD group and 23 in the UD group were either medication-naive or had been unmedicated for at least five months. The remaining patients with $\mathrm{BD}$ were receiving antidepressants (duloxetine or paroxetine), and/or mood stabilizers (lithium, sodium valproate), and/or atypical antipsychotic medications (olanzapine or risperidone). The remaining patients with UD were receiving antidepressants (duloxetine or paroxetine). The treatment duration for these $\mathrm{BD}$ and $\mathrm{UD}$ 
patients was no more than six weeks.

Forty healthy controls (HC) were recruited by local advertisements. They were carefully screened through a diagnostic interview, the Structured Clinical Interview for DSM-IV Nonpatient Edition (SCID-NP), to rule out the presence of current or past history of substance abuse/dependence or any psychiatric illness in self or in first-degree relatives. Further exclusion criteria for HC were any history of cerebrovascular disease, past head injury, epilepsy, migraine, hypertension, diabetes, and other types of disorders potentially affecting the central nervous system. All participants were right-handed and were submitted to MRI scanning within $48 \mathrm{~h}$ of initial contact. This study was conducted in accordance with the Declaration of Helsinki (1989) and was approved by the Ethics Committee of the First Affiliated Hospital of Jinan University, China. All participants signed informed consent form after a full written and verbal explanation of the study. Two senior clinical psychiatrists confirmed that all subjects had the ability to consent to participate in the examination.

\section{MR techniques}

Experiments were conducted on a 3 Tesla MR system (Discovery MR 750 System, GE Healthcare, Milwaukee, WI, USA) with an 8-channel phased array head coil. Subjects were scanned in a supine, head-first position with symmetrically placed cushions on both sides of the head to decrease motion.

The DKI experiments were performed using a twice-refocused-spin-echo (TRSE) diffusion sequence with 15 different diffusion encoding directions using an optimized sampling strategy. For each direction, six b-values $(b=0,500,1000,1500$, 2000, $2500 \mathrm{~s} / \mathrm{mm}^{2}$ ) were used. Other imaging parameters were: $\mathrm{TR}=4500 \mathrm{~ms}, \mathrm{TE}=$ minimum, field of view $(\mathrm{FOV})=$ $256 \times 256 \mathrm{~mm}^{2}$, matrix $=128 \times 128$, parallel imaging factor of 2 with $24 \mathrm{k}$-lines used as references, number of averages $=2$, slice thickness $=2 \mathrm{~mm}$, voxel size $=2 \times 2 \times 2 \mathrm{~mm}^{3}$. The total scan duration for the DKI sequence was $12 \mathrm{~min} 5 \mathrm{sec}$. 3D ASL was performed by use of a pseudocontinuous arterial spin labeling (pCASL) period of $1500 \mathrm{~ms}$ with a post labeling delay time of $1525 \mathrm{~ms}$. TR=4632 ms, TE $=10.5 \mathrm{~ms}, \mathrm{FOV}=24 \times 24 \mathrm{~cm}$, slice thickness $=4 \mathrm{~mm}$. Whole-brain images were obtained with an interleaved 3D stack of spirals fast spin echo (FSE) sequence and background suppression. Multiarm spiral imaging was used, with 8 arms and 1024 points acquired on each arm. A high level of background suppression was achieved by use of 4 separate inversion pulses spaced around the pseudocontinuous labeling pulse. The entire process took $4 \mathrm{~min} 29 \mathrm{sec}$ to complete which included proton attenuation. In addition, a three dimensional brain volume imaging (3D BRAVO) sequence covering the whole brain was used for structural data acquisition with: TR/TE=8.2 $\mathrm{msec} / 3.2 \mathrm{msec}$, slice thick- ness $=1.0 \mathrm{~mm}$, gap $=0 \mathrm{~mm}$, matrix $=256 \times 256, \mathrm{FOV}=24 \times 24$ $\mathrm{cm}, \mathrm{NEX}=1$, flip angle $=12^{\circ}$, bandwidth $=31.25 \mathrm{~Hz}$, acquisition time $=3$ min $45 \mathrm{sec}$. Routine MRI examination images were also collected for excluding anatomic abnormality, such as T1 weighted, T2 weighted, DWI, and T2/fluid-attenuated inversion recovery (FLAIR) images. All participants were found, by two experienced radiologists, to have no abnormalities on routine MRI.

\section{Data processing}

The data were transferred to a dedicated workstation (General Electric Advantage Workstation 4.5) where the DKI and 3D ASL data were post-processed using Functool software version 9.4.05a. The Functool program corrected echo planar imaging (EPI) distortion and eddy current. The diffusion and kurtosis tensors were calculated using the DKI model described previously by Jensen et al. ${ }^{26,28}$ which embedded in the Functool software. The key relationship is

$$
\operatorname{In}[S(b)]=\operatorname{In}[S(0)]-b D_{a p p}+\frac{1}{6} b^{2} D_{a p p}^{2} K_{a p p}+O\left(b^{3}\right)
$$

Where $\mathrm{S}(\mathrm{b})$ is the signal intensity at the echo time, $D_{\text {app }}$ is the apparent diffusion coefficient, and $K_{a p p}$ is the apparent diffusional kurtosis. The parameter $b$ is given by the usual expression $b=(\gamma \delta g)^{2}(\Delta-\delta / 3)$, where $\gamma$ is the proton gyromagnetic ratio. In carrying out the expansion of equation [1], it is assumed that $b$ is changed by varying the gradient strength $g$ with the timing parameters $\delta$ and $\Delta$ being kept fixed. Just as $D_{a p p}$ is an estimate for the diffusion coefficient in the direction parallel to the orientation of diffusion sensitizing gradients, $K_{a p p}$ is an estimate for the diffusional kurtosis in this same direction. ${ }^{28}$ With our DKI protocol, we obtained parametric maps related to diffusional kurtosis: $\mathrm{MK}, \mathrm{Ka}$ and $\mathrm{Kr}$. Conventional DTIbased metrics were also derived, including FA, MD, Da and Dr. Details for the computation of these metrics have been described previously. ${ }^{25,26}$

The kinetic model for 3D ASL proposed by Alsop and Detre was used in this study. ${ }^{39}$ Besides, we included a term for the finite labeling duration ${ }^{40}$ and correct the incomplete recovery of the tissue signal in the reference image due to the saturation performed $t_{\text {sat }}(2000 \mathrm{~ms})$ before imaging. ${ }^{41}$ The quantitative $\mathrm{CBF}$ maps were generated using the following equation $^{41,42}$ :

$$
C B F=\frac{\lambda\left(1-e^{-\frac{l_{s a t}}{T_{1 g}}}\right)}{2 \alpha T_{1 b}\left(1-e^{-\frac{\tau}{T_{1 b}}}\right)} \frac{\Delta S}{S_{o}} e^{\frac{\omega}{T_{1 b}}}
$$

Where CBF is the cerebral blood flow; $T_{1 \mathrm{~b}}$ is the $T 1$ relaxation time of the blood $(1600 \mathrm{~ms})$ in $3.0 \mathrm{~T}, T_{1 \mathrm{~g}}$ represents the 
$T 1$ relaxation time of the gray matter $(1200 \mathrm{~ms})$ in $3.0 \mathrm{~T}$, $t_{\text {sat }}$ is the duration time of saturation pulse performed before imaging (2000 ms), $\alpha$ is the labeling efficiency (0.8), $\lambda$ is brain/blood partition coefficient $(0.9), \tau$ is the labeling duration (1500 $\mathrm{ms}), \omega$ is the postlabeling delay time (1525 ms). The inversion slab is $22 \mathrm{~mm}$ below the acquired volume. Resolution of pCASL data is $3.7 \mathrm{~mm}$ (FOV: $24 \mathrm{~cm}$, reconstructed matrix: $64 \times 64)$.

Regions of interest (ROIs) drawing methods were made based on Xueying et al. ${ }^{11}$ and He et al.s ${ }^{12}$ ROIs analysis and our previous study, ${ }^{36}$ and determined by two independent neuroradiologists (LP. Zhao and Y. Wang, with 5 and 9 years of experience, respectively) who were blinded to the patient or control status. For each subject, DKI, 3D ASL and 3D BRAVO can fused with each other. ROIs manually placed in the bilateral caudate nucleus head, putamen and thalamus on the maximum level of these structures. Considering the relatively low resolution of the DKI and CBF maps, we selected high resolution 3D BRAVO images to fuse with the DKI and CBF maps as the reference image. The $b_{0}$ image (which is essentially a $\mathrm{T}_{2}$-weighted image) for the slices with bilateral caudate nucleus head, putamen and thalamus and the high resolution 3D BRAVO images illustrate how and where the ROIs were drawn in these structures (Supplementary Figure 1 in the online-only Data Supplement). Separate ROIs were drawn carefully in the bilateral caudate nucleus head, putamen and thalamus, which are hypointense on the $\mathrm{b}_{0}$ images firstly, and then the 3D BRAVO images were used to minimize variation and better delineate the borders of the ROIs against surrounding structures (i.e., internal capsule and external capsule), which appear hyperintense on the 3D BRAVO images. For each subject, ROI sizes were identical in the left and right hemispheres by using the mirror symmetry tools from the Functool software. However, the size of caudate nucleus, putamen and thalamus differed among the subjects, and thus the ROIs could not be the same. The areas in the caudate nucleus, putamen and thalamus ranged from 101 to $171 \mathrm{~mm}^{2}$, from 232 to $495 \mathrm{~mm}^{2}$ and from 424 to $598 \mathrm{~mm}^{2}$ respectively. Major vascular structures and artefacts were avoided in placing the ROIs. All these ROIs of DKI parameters were then transferred to the maps of FA, MD, Da, Dr, MK, Ka, and Kr (Supplementary Figure 2 in the online-only Data Supplement) while the ROIs of 3D ASL were transferred to the maps of CBF (Supplementary Figure $2 \mathrm{H}$ in the online-only Data Supplement) for measurement.

\section{Statistical analysis}

Statistical analyses were performed using SPSS for Windows software, version 17.0 (SPSS Inc., Chicago, IL, USA). Distributions of age and years of education between the three groups were compared with one-way analysis of variance (ANOVA). Chi-square test was used to compare gender distributions. Multivariate analysis of variance (MANOVA) with Bonferroni's pairwise comparisons tests was performed to compare the DKI parameters and CBF values of all the three ROIs among $\mathrm{BD}, \mathrm{UD}$ and $\mathrm{HC}$ groups respectively in the left and right hemispheres and adjusted for age, with sex and group as fixed factors. Then, the Receiver operating characteristic (ROC) curve was drawn for the significant parameters from MANOVA to evaluate the predictive value of these parameters on $\mathrm{BD}$ and UD diagnosis and to determine the best cut-off point to discriminate patients with BD or UD from HC. Pearson's correlation coefficients were used to correlate the clinical variables for the patients to their measured DKI and 3D ASL parameters. Inter-rater reliability was assessed using the Intra-class correlation coefficient (ICC) analysis. A p-value of less than 0.05 was considered statistically significant.

\section{RESULTS}

\section{Demographic result}

Table 1 shows the demographics and clinical data of all study participants. There was no significant difference between the groups of BD, UD and $\mathrm{HC}$ in age, gender and education levels of the subjects recruited for this study. The two depressed groups were matched for depression severity, illness duration, and illness onset age.

\section{Inter-rater reliability analysis}

ICC analysis showed a significantly high a value which was all greater than 0.70 in the three ROIs (i.e., caudate nucleus head, putamen, and thalamus), and ICC value was close to 1 $(p<0.05)$. Hence, all measurements were regarded as reliable among different raters (Table 2). Therefore, averaged values were used for subsequent statistical analyses.

\section{Group differences of the parameters generated from DKI}

Table 3 shows the DKI parameters in BD, UD patients and HC participants (Figure 1 and Supplementary Figure 3 in the online-only Data Supplement). In the thalamus, MANOVArevealed significant differences in Dr in the left $(p=0.048)$, however, pairwise comparisons showed no significant differences between them. In the putamen, MANOVA revealed significant differences in MK (right: $\mathrm{p}=0.038$ ) and Ka (left: $\mathrm{p}=0.009$, right: $\mathrm{p}=0.031$ ) among the three groups. Pairwise comparisons only revealed the UD group showed significantly increased Ka compared with HC $(\mathrm{p}=0.039)$ and BD $(\mathrm{p}=$ 0.026 ) group in left putamen. In the caudate nucleus head, MANOVA revealed significant differences in $\mathrm{Ka}(\mathrm{p}=0.034)$ 
Table 1. The demographic characteristics of the subjects

\begin{tabular}{|c|c|c|c|c|c|}
\hline Parameters & $\mathrm{BD}(\mathrm{N}=35)$ & $\mathrm{UD}(\mathrm{N}=30)$ & $\mathrm{HC}(\mathrm{N}=40)$ & \multicolumn{2}{|c|}{ Statistic } \\
\hline Gender (male/female) & $18 / 17$ & $13 / 17$ & $21 / 19$ & $\chi^{2}=0.652$ & $\mathrm{p}=0.722$ \\
\hline Age (years) & $30.31(10.07)$ & $33.9(9.28)$ & $32.50(12.21)$ & $\mathrm{F}=0.917$ & $\mathrm{p}=0.403$ \\
\hline Education (years) & $13.31(3.20)$ & $12.94(3.53)$ & $14.43(3.34)$ & $\mathrm{F}=1.857$ & $\mathrm{p}=0.161$ \\
\hline Duration of illness (months) & $41.77(39.56)$ & $34.71(52.28)$ & N/A & $\mathrm{t}=0.643$ & $\mathrm{p}=0.522$ \\
\hline Age of illness onset (years) & $25.78(9.11)$ & $29.66(12.72)$ & $\mathrm{N} / \mathrm{A}$ & $\mathrm{t}=1.444$ & $\mathrm{p}=0.154$ \\
\hline HDRS-24 score (points) & $25.73(5.36)$ & $24.08(6.14)$ & N/A & $t=-1.164$ & $\mathrm{p}=0.249$ \\
\hline YMRS score (points) & $2.68(1.15)$ & $2.15(1.22)$ & N/A & $t=-1.791$ & $\mathrm{p}=0.078$ \\
\hline Number of past manic/depressive episodes & $2.02(2.01)$ & $3.26(6.07)$ & $\mathrm{N} / \mathrm{A}$ & $\mathrm{N}$ & $\mathrm{N}$ \\
\hline
\end{tabular}

Values are reported as mean (standard deviation), except for gender. BD: bipolar disorder, UD: unipolar depression, HC: healthy controls, HDRS: Hamilton Depression Rating Scale, YMRS: YoungMania Rating Scale

Table 2. Inter-rater reliability of DKI and 3D ASL parameters in the basal ganglia and thalamus

\begin{tabular}{|c|c|c|c|c|c|c|c|c|c|c|c|c|c|c|c|c|}
\hline \multirow{2}{*}{$\begin{array}{l}\text { ROIs and } \\
\text { parameters }\end{array}$} & \multicolumn{2}{|c|}{ MK } & \multicolumn{2}{|c|}{$\mathrm{Ka}$} & \multicolumn{2}{|c|}{$\mathrm{Kr}$} & \multicolumn{2}{|c|}{ FA } & \multicolumn{2}{|c|}{ MD } & \multicolumn{2}{|c|}{$\mathrm{Da}$} & \multicolumn{2}{|c|}{ Dr } & \multicolumn{2}{|c|}{$\mathrm{CBF}$} \\
\hline & $\mathrm{L}$ & $\mathrm{R}$ & $\mathrm{L}$ & $\mathrm{R}$ & $\mathrm{L}$ & $\mathrm{R}$ & $\mathrm{L}$ & $\mathrm{R}$ & $\mathrm{L}$ & $\mathrm{R}$ & $\mathrm{L}$ & $\mathrm{R}$ & $\mathrm{L}$ & $\mathrm{R}$ & $\mathrm{L}$ & $\mathrm{R}$ \\
\hline \multicolumn{17}{|l|}{ Caudate } \\
\hline$\alpha$ & 0.891 & 0.917 & 0.959 & 0.902 & 0.865 & 0.884 & 0.997 & 0.992 & 0.916 & 0.951 & 0.880 & 0.961 & 0.888 & 0.923 & 0.969 & 0.975 \\
\hline ICC & 0.804 & 0.846 & 0.922 & 0.821 & 0.762 & 0.792 & 0.993 & 0.985 & 0.844 & 0.906 & 0.786 & 0.925 & 0.798 & 0.856 & 0.941 & 0.951 \\
\hline $\mathrm{p}$ & 0.000 & 0.000 & 0.000 & 0.000 & 0.000 & 0.000 & 0.000 & 0.000 & 0.000 & 0.000 & 0.000 & 0.000 & 0.000 & 0.000 & 0.000 & 0.000 \\
\hline \multicolumn{17}{|l|}{ Putamen } \\
\hline$a$ & 0.881 & 0.942 & 0.958 & 0.955 & 0.863 & 0.933 & 0.903 & 0.865 & 0.939 & 0.951 & 0.878 & 0.841 & 0.952 & 0.945 & 0.987 & 0.986 \\
\hline ICC & 0.788 & 0.891 & 0.919 & 0.914 & 0.759 & 0.875 & 0.823 & 0.762 & 0.884 & 0.907 & 0.783 & 0.725 & 0.908 & 0.896 & 0.974 & 0.972 \\
\hline $\mathrm{p}$ & 0.000 & 0.000 & 0.000 & 0.000 & 0.000 & 0.000 & 0.000 & 0.000 & 0.000 & 0.000 & 0.000 & 0.000 & 0.000 & 0.000 & 0.000 & 0.000 \\
\hline \multicolumn{17}{|l|}{ Thalamus } \\
\hline$\alpha$ & 0.928 & 0.915 & 0.879 & 0.950 & 0.956 & 0.933 & 0.967 & 0.966 & 0.925 & 0.906 & 0.966 & 0.964 & 0.970 & 0.962 & 0.871 & 0.875 \\
\hline ICC & 0.866 & 0.843 & 0.783 & 0.904 & 0.915 & 0.875 & 0.936 & 0.934 & 0.860 & 0.829 & 0.933 & 0.931 & 0.942 & 0.926 & 0.772 & 0.778 \\
\hline $\mathrm{p}$ & 0.000 & 0.000 & 0.000 & 0.000 & 0.000 & 0.000 & 0.000 & 0.000 & 0.000 & 0.000 & 0.000 & 0.000 & 0.000 & 0.000 & 0.000 & 0.000 \\
\hline
\end{tabular}

MK: mean kurtosis, Ka: axial kurtosis, Kr: radial kurtosis, FA: fractionalanisotropy, MD: mean diffusivity, Da: axial diffusivity, Dr: radial diffusivity, L: left, R: right, $\alpha$ : Cronbach's alpha, ICC: intra-classcorrelation coefficient, ROIs: regions of interest, DKI: diffusional kurtosis imaging, 3D ASL: three dimensional arterial spin labeling

and $\mathrm{Kr}(\mathrm{p}=0.002)$ in the left and $\mathrm{MD}(\mathrm{p}=0.026)$ in the right caudate nucleus head among the three groups. Pairwise comparisons showed significantly increased $\mathrm{Ka}(\mathrm{p}=0.033)$ in UD compared with $\mathrm{HC}$, and significantly increased $\mathrm{Kr}$ both in $\mathrm{BD}$ $(\mathrm{p}=0.014)$ and $\mathrm{UD}(\mathrm{p}=0.005)$ compared with HC in the left. In addition, $\mathrm{MD}$ values were significantly lower in $\mathrm{UD}(\mathrm{p}=$ 0.021 ) compared with $\mathrm{BD}$ in the right caudate nucleus. No significant changes in the remaining DKI parameters were observed in the three ROIs among the three groups.

\section{Group differences of CBF}

Table 4 shows the results of CBF in the three groups (Figure 2 and Supplementary Figure 4 in the online-only Data Supplement). MANOVA revealed significant differences in the left caudate nucleus head and putamen between the three groups. Pairwise comparisons showed significantly decreased CBF in UD compared with $\mathrm{HC}$ in the left caudate nucleus head $(\mathrm{p}=$
0.033 ) and putamen ( $\mathrm{p}=0.045)$. No significant changes in the rest of the CBF were observed in these ROIs among the three groups.

\section{ROC analysis}

As shown in Table 5 and Supplementary Figure 5 (in the online-only Data Supplement), the predictive value for UD from $\mathrm{HC}$ in the left caudate nucleus head was $\mathrm{Ka}$ (AUC= 0.657 , sensitivity $=73.1 \%$, specificity $=51.2 \%), \mathrm{Kr}(\mathrm{AUC}=0.726$, sensitivity $=84.6 \%$, specificity $=60.5 \%$ ) and $\mathrm{CBF}(\mathrm{AUC}=0.657$, sensitivity $=70.0 \%$, specificity $=57.1 \%$ ); in the left putamen was $\mathrm{Ka}$ ( $\mathrm{AUC}=0.685$, sensitivity $=70.8 \%$, specificity $=56.1 \%$ ) and $\mathrm{CBF}$ (AUC=0.676, sensitivity $=73.3 \%$, specificity $=57.1 \%$ ). The predictive value for $\mathrm{BD}$ from $\mathrm{HC}$ in the left caudate nucleus head was $\mathrm{Kr}$ (AUC $=0.664$, sensitivity $=74.4 \%$, specificity $=60.5 \%)$. 
Table 3. Comparison of the DKI parameters of subcortical gray matters in patients with BD, UD and HC subjects

\begin{tabular}{|c|c|c|c|c|c|c|c|c|c|}
\hline \multirow{2}{*}{$\begin{array}{l}\text { ROIs and } \\
\text { parameters }\end{array}$} & \multirow{2}{*}{ Side } & \multicolumn{3}{|c|}{ Group } & \multirow{2}{*}{ F-value } & \multirow{2}{*}{$\begin{array}{l}\text { MANOVA } \\
\text { p value }\end{array}$} & \multicolumn{3}{|c|}{ Pairwise comparison $\mathrm{p}$ value } \\
\hline & & $\mathrm{BD}(\mathrm{N}=35)$ & $\mathrm{UD}(\mathrm{N}=30)$ & $\mathrm{HC}(\mathrm{N}=40)$ & & & BD vs. UD & UD vs. HC & $\mathrm{BD}$ vs. $\mathrm{HC}$ \\
\hline \multicolumn{10}{|c|}{ Caudate nucleus head } \\
\hline \multirow[t]{2}{*}{ MK } & Left & $0.52(0.05)$ & $0.51(0.04)$ & $0.53(0.05)$ & 1.036 & 0.358 & 0.995 & 0.424 & 0.596 \\
\hline & Right & $0.54(0.06)$ & $0.54(0.06)$ & $0.54(0.06)$ & 0.068 & 0.934 & 0.994 & 0.976 & 0.998 \\
\hline \multirow[t]{2}{*}{$\mathrm{Ka}$} & Left & $0.61(0.05)$ & $0.63(0.06)$ & $0.60(0.05)$ & 3.479 & $0.034^{\dagger}$ & 0.695 & $0.033^{*}$ & 0.342 \\
\hline & Right & $0.61(0.04)$ & $0.59(0.05)$ & $0.60(0.05)$ & 1.327 & 0.270 & 0.510 & 1.000 & 0.514 \\
\hline \multirow[t]{2}{*}{$\mathrm{Kr}$} & Left & $0.52(0.07)$ & $0.50(0.05)$ & $0.56(0.07)$ & 6.632 & $0.002^{\dagger}$ & 1.000 & $0.005^{*}$ & $0.014^{*}$ \\
\hline & Right & $0.55(0.10)$ & $0.57(0.10)$ & $0.60(0.09)$ & 2.093 & 0.128 & 0.948 & 0.513 & 0.139 \\
\hline \multirow[t]{2}{*}{ FA } & Left & $0.19(0.03)$ & $0.20(0.03)$ & $0.19(0.02)$ & 0.371 & 0.691 & 0.864 & 0.999 & 0.851 \\
\hline & Right & $0.19(0.04)$ & $0.20(0.03)$ & $0.20(0.03)$ & 0.379 & 0.685 & 0.801 & 0.996 & 0.885 \\
\hline \multirow[t]{2}{*}{$\mathrm{MD}$} & Left & $0.90(0.05)$ & $0.89(0.06)$ & $0.89(0.02)$ & 0.753 & 0.473 & 0.675 & 0.975 & 0.731 \\
\hline & Right & $0.93(0.05)$ & $0.90(0.04)$ & $0.91(0.05)$ & 3.779 & $0.026^{\dagger}$ & $0.021^{*}$ & 0.768 & 0.164 \\
\hline \multirow[t]{2}{*}{$\mathrm{Da}$} & Left & $1.07(0.06)$ & $1.06(0.05)$ & $1.07(0.05)$ & 0.389 & 0.678 & 0.920 & 0.732 & 0.982 \\
\hline & Right & $1.11(0.08)$ & $1.05(0.02)$ & $1.11(0.06)$ & 2.791 & 0.066 & 0.355 & 0.386 & 0.996 \\
\hline \multirow[t]{2}{*}{ Dr } & Left & $0.81(0.05)$ & $0.80(0.06)$ & $0.80(0.04)$ & 0.365 & 0.695 & 0.893 & 0.999 & 0.832 \\
\hline & Right & $0.83(0.06)$ & $0.80(0.04)$ & $0.82(0.04)$ & 2.705 & 0.071 & 0.065 & 0.480 & 0.470 \\
\hline \multicolumn{10}{|l|}{ Putamen } \\
\hline \multirow[t]{2}{*}{ MK } & Left & $0.60(0.05)$ & $0.60(0.03)$ & $0.60(0.04)$ & 0.012 & 0.988 & 0.998 & 1.000 & 1.000 \\
\hline & Right & $0.61(0.05)$ & $0.65(0.06)$ & $0.61(0.04)$ & 3.379 & $0.038^{\dagger}$ & 0.052 & 0.076 & 1.000 \\
\hline \multirow[t]{2}{*}{$\mathrm{Ka}$} & Left & $0.70(0.03)$ & $0.73(0.04)$ & $0.71(0.03)$ & 4.966 & $0.009^{\dagger}$ & $0.026^{*}$ & $0.039^{*}$ & 0.996 \\
\hline & Right & $0.70(0.03)$ & $0.72(0.05)$ & $0.70(0.03)$ & 3.595 & $0.031^{\dagger}$ & 0.105 & 0.111 & 1.000 \\
\hline \multirow[t]{2}{*}{$\mathrm{Kr}$} & Left & $0.56(0.08)$ & $0.56(0.07)$ & $0.56(0.07)$ & 0.053 & 0.949 & 0.982 & 0.997 & 0.998 \\
\hline & Right & $0.59(0.09)$ & $0.62(0.09)$ & $0.59(0.08)$ & 1.401 & 0.251 & 0.507 & 0.369 & 0.993 \\
\hline \multirow[t]{2}{*}{ FA } & Left & $0.22(0.03)$ & $0.22(0.03)$ & $0.22(0.03)$ & 0.973 & 0.381 & 0.774 & 0.996 & 0.419 \\
\hline & Right & $0.22(0.03)$ & $0.22(0.03)$ & $0.22(0.03)$ & 0.741 & 0.479 & 0.518 & 0.902 & 0.889 \\
\hline \multirow[t]{2}{*}{$\mathrm{MD}$} & Left & $0.84(0.03)$ & $0.84(0.03)$ & $0.84(0.03)$ & 0.255 & 0.776 & 0.996 & 0.891 & 0.934 \\
\hline & Right & $0.86(0.03)$ & $0.88(0.04)$ & $0.86(0.02)$ & 2.924 & 0.058 & 0.174 & 0.145 & 1.000 \\
\hline \multirow[t]{2}{*}{$\mathrm{Da}$} & Left & $1.03(0.05)$ & $1.04(0.04)$ & $1.04(0.05)$ & 0.602 & 0.550 & 0.773 & 1.000 & 0.700 \\
\hline & Right & $1.05(0.05)$ & $1.08(0.06)$ & $1.06(0.05)$ & 2.476 & 0.089 & 0.134 & 0.415 & 0.774 \\
\hline \multirow[t]{2}{*}{$\mathrm{Dr}$} & Left & $0.74(0.03)$ & $0.74(0.03)$ & $0.74(0.02)$ & 0.435 & 0.648 & 0.997 & 0.764 & 0.844 \\
\hline & Right & $0.76(0.04)$ & $0.77(0.03)$ & $0.76(0.03)$ & 1.791 & 0.172 & 0.307 & 0.229 & 1.000 \\
\hline Thalamus & & & & & & & & & \\
\hline MK & Left & $0.71(0.04)$ & $0.73(0.03)$ & $0.72(0.04)$ & 2.754 & 0.068 & 0.072 & 0.680 & 0.357 \\
\hline & Right & $0.71(0.04)$ & $0.73(0.04)$ & $0.72(0.04)$ & 0.238 & 0.792 & 0.876 & 0.951 & 0.986 \\
\hline $\mathrm{Ka}$ & Left & $0.67(0.04)$ & $0.68(0.03)$ & $0.67(0.04)$ & 0.718 & 0.490 & 0.769 & 0.530 & 0.978 \\
\hline & Right & $0.67(0.04)$ & $0.67(0.04)$ & $0.66(0.03)$ & 0.741 & 0.479 & 1.000 & 0.783 & 0.554 \\
\hline $\mathrm{Kr}$ & Left & $0.77(0.08)$ & $0.80(0.06)$ & $0.78(0.06)$ & 0.824 & 0.442 & 0.501 & 0.899 & 0.826 \\
\hline & Right & $0.79(0.08)$ & $0.81(0.06)$ & $0.80(0.06)$ & 0.095 & 0.909 & 0.982 & 1.000 & 0.974 \\
\hline FA & Left & $0.31(0.03)$ & $0.29(0.03)$ & $0.31(0.03)$ & 2.531 & 0.084 & 0.072 & 0.243 & 0.873 \\
\hline & Right & $0.31(0.03)$ & $0.29(0.03)$ & $0.30(0.03)$ & 1.801 & 0.169 & 0.201 & 0.923 & 0.439 \\
\hline $\mathrm{MD}$ & Left & $0.90(0.02)$ & $0.91(0.03)$ & $0.90(0.03)$ & 2.529 & 0.085 & 0.521 & 0.123 & 0.584 \\
\hline & Right & $0.90(0.03)$ & $0.91(0.03)$ & $0.90(0.03)$ & 0.875 & 0.420 & 0.620 & 0.453 & 0.986 \\
\hline $\mathrm{Da}$ & Left & $1.19(0.06)$ & $1.19(0.04)$ & $1.18(0.03)$ & 0.204 & 0.816 & 0.993 & 0.976 & 0.897 \\
\hline & Right & $1.20(0.07)$ & $1.19(0.04)$ & $1.18(0.04)$ & 0.712 & 0.493 & 0.974 & 0.793 & 0.585 \\
\hline
\end{tabular}


Table 3. Comparison of the DKI parameters of subcortical gray matters in patients with BD, UD and HC subjects (continued)

\begin{tabular}{|c|c|c|c|c|c|c|c|c|c|}
\hline \multirow{2}{*}{$\begin{array}{c}\text { ROIs and } \\
\text { parameters }\end{array}$} & \multirow{2}{*}{ Side } & \multicolumn{3}{|c|}{ Group } & \multirow{2}{*}{ F-value } & \multirow{2}{*}{$\begin{array}{c}\text { MANOVA } \\
\text { p value }\end{array}$} & \multicolumn{3}{|c|}{ Pairwise comparison $\mathrm{p}$ value } \\
\hline & & $\mathrm{BD}(\mathrm{N}=35)$ & $\mathrm{UD}(\mathrm{N}=30)$ & $\mathrm{HC}(\mathrm{N}=40)$ & & & BD vs. UD & UD vs. HC & BD vs. HC \\
\hline \multirow[t]{2}{*}{ Dr } & Left & $0.75(0.03)$ & $0.77(0.03)$ & $0.75(0.03)$ & 3.132 & $0.048^{\dagger}$ & 0.055 & 0.096 & 0.984 \\
\hline & Right & $0.75(0.04)$ & $0.77(0.03)$ & $0.76(0.04)$ & 1.730 & 0.182 & 0.146 & 0.368 & 0.937 \\
\hline
\end{tabular}

Diffusional kurtosis metrics estimates reported as mean (standard deviation) in caudate nucleus head, putamen and thalamus. The units for $\mathrm{MD}, \mathrm{Da}$ and Dr are all $\mu \mathrm{m}^{2} / \mathrm{ms}$; MK, Ka, Kr and FA are dimensionless parameters. *the group difference remained significant after applying Bonferroni correction, ${ }^{\dagger}$ multivariate analysis of variance (MANOVA) with significant p-values with. DKI: diffusional kurtosis imaging, MK: mean kurtosis, Ka: axial kurtosis, Kr: radial kurtosis, FA: fractional anisotropy, MD: mean diffusivity, Da: axial diffusivity, Dr: radial diffusivity, BD: bipolar disorder, UD: unipolar depression, HC: healthy controls
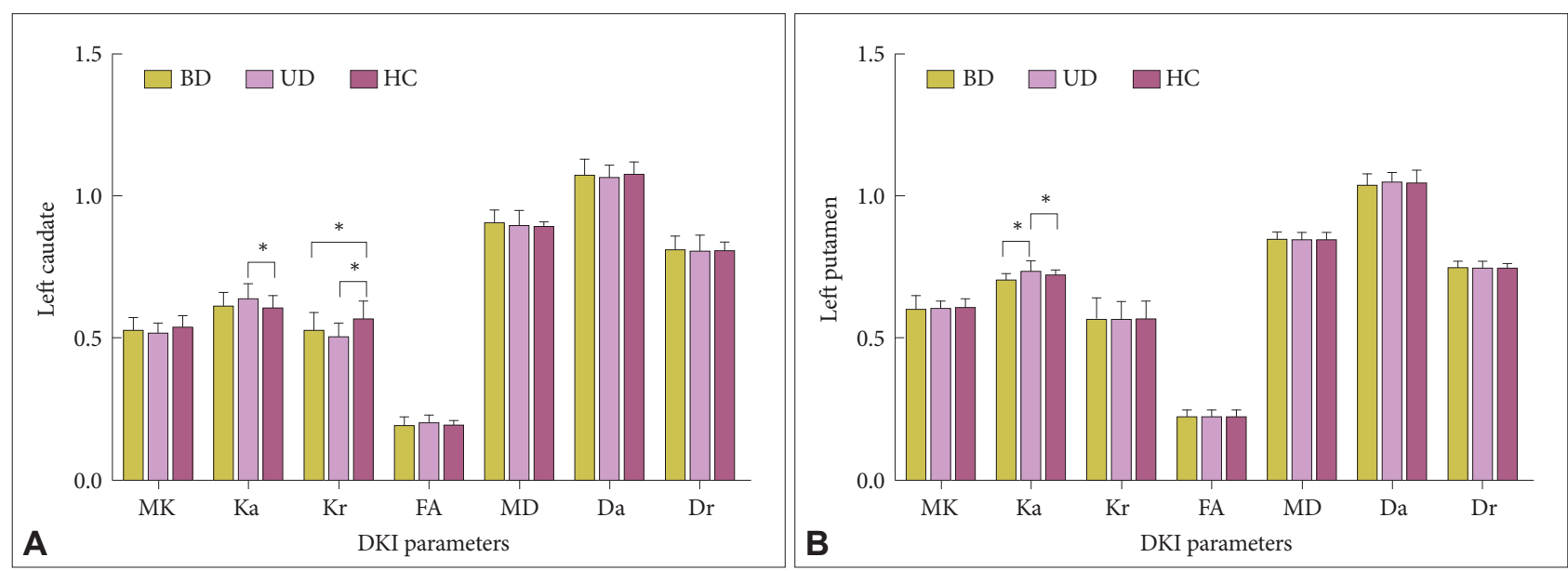

Figure 1. Group differences of the DKI parameters in the left caudate nucleus head (A) and putamen (B) respectively. ${ }^{*} p<0.05$. The units for $\mathrm{MD}$, $\mathrm{Da}$ and $\mathrm{Dr}$ are all $\mu \mathrm{m}^{2} / \mathrm{ms}$; MK, Ka, $\mathrm{Kr}$ and FA are dimensionless parameters. DKI: diffusional kurtosis imaging, MK: mean kurtosis, Ka: axial kurtosis, Kr: radial kurtosis, FA: fractional anisotropy, MD: mean diffusivity, Da: axial diffusivity, Dr: radial diffusivity, BD: bipolar disorder, UD: unipolar depression, HC: healthy controls.

Table 4. Comparison of the CBF from 3D ASL of subcortical gray matters in patient with BD, UD and healthy controls

\begin{tabular}{|c|c|c|c|c|c|c|c|c|c|}
\hline \multirow{2}{*}{$\begin{array}{l}\text { ROIs and } \\
\text { parameters }\end{array}$} & \multirow{2}{*}{ Side } & \multicolumn{3}{|c|}{ Group } & \multirow{2}{*}{ F-value } & \multirow{2}{*}{$\begin{array}{l}\text { Manova } \\
\text { p value }\end{array}$} & \multicolumn{3}{|c|}{ Pairwise comparison $\mathrm{p}$ value } \\
\hline & & $\mathrm{BD}(\mathrm{N}=35)$ & $\mathrm{UD}(\mathrm{N}=30)$ & $\mathrm{HC}(\mathrm{N}=40)$ & & & BD vs. UD & UD vs. HC & $\mathrm{BD}$ vs. $\mathrm{HC}$ \\
\hline \multicolumn{10}{|c|}{ Caudate nucleus head } \\
\hline \multirow[t]{2}{*}{$\mathrm{CBF}$} & Left & $54.82(6.73)$ & $51.93(7.54)$ & $56.81(9.05)$ & 3.460 & $0.035^{\dagger}$ & 0.597 & $0.033^{*}$ & 0.438 \\
\hline & Right & $57.27(8.44)$ & $54.86(7.12)$ & $59.00(9.42)$ & 2.361 & 0.099 & 1.000 & 0.134 & 0.395 \\
\hline \multicolumn{10}{|l|}{ Putamen } \\
\hline \multirow[t]{2}{*}{$\mathrm{CBF}$} & Left & $51.03(7.31)$ & $47.15(7.57)$ & $51.50(7.57)$ & 3.462 & $0.035^{\dagger}$ & 0.085 & $0.045^{*}$ & 1.000 \\
\hline & Right & $52.14(7.74)$ & $49.24(5.90)$ & $53.52(8.25)$ & 2.933 & 0.057 & 0.308 & 0.052 & 1.000 \\
\hline \multicolumn{10}{|l|}{ Thalamus } \\
\hline \multirow[t]{2}{*}{$\mathrm{CBF}$} & Left & $52.26(8.95)$ & $52.46(8.37)$ & $55.71(8.90)$ & 2.041 & 0.134 & 1.000 & 0.357 & 0.197 \\
\hline & Right & $52.36(9.17)$ & $52.58(8.14)$ & $56.57(9.32)$ & 2.929 & 0.057 & 1.000 & 0.185 & 0.084 \\
\hline
\end{tabular}

CBF generated from 3D ASL are reported as mean (standard deviation) in caudate nucleus head, putamen and thalamus. The unit for CBF is $\mathrm{mL} / 100 \mathrm{~g} / \mathrm{min}$. *the group difference remained significant after applying Bonferroni correction, ${ }^{\dagger}$ multivariate analysis of variance (MANOVA) with significant p-values with. CBF: cerebral blood flow, BD: bipolar disorder, UD: unipolar depression, HC: healthy controls, 3D ASL: three dimensional arterial spin labeling

\section{Correlations between DKI/CBF parameters and HDRS-24 score/illness duration}

There were no significant correlations between the DKI and 3D ASL parameters in the three ROIs and the clinical variables (HDRS scores, illness duration) in the patients with ei- ther BD or UD.

\section{DISCUSSION}

The current study, to our knowledge, is the first study to di- 
rectly compare the difference in the microstructural organization and perfusion of basal ganglia and thalamus in $\mathrm{BD}$ and UD depressed adults and healthy subjects by using DKI and 3D ASL. The two disorders showed similarities and differences in the microstructural and perfusional changes in the basal ganglia. This study has several principal findings. First, patients with UD exhibited microstructural abnormalities and CBF reduction in the left putamen and caudate nucleus head compared with HC subjects. Second, patients with BD exhibited microstructural abnormalities only in the left caudate nucleus head compared with HC subjects. Last, patients with BD showed significantly lower Ka values in right putamen, higher MD values in the right caudate nucleus head compared with patients with UD.

In the basal ganglia, we observed increased $\mathrm{Ka}$ and decreased $\mathrm{Kr}$ in the left caudate nucleus, increased $\mathrm{Ka}$ in the left putamen in UD group compared with HC subjects. We also

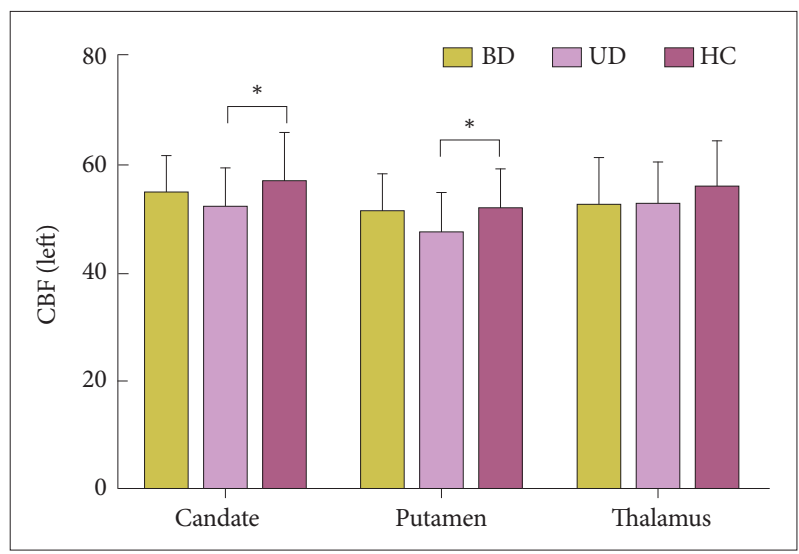

Figure 2. Group differences of $\mathrm{CBF}$ in the left caudate nucleus head, putamen and thalamus respectively. ${ }^{*} p<0.05$. CBF: cerebral blood flow, BD: bipolar disorder, UD: unipolar depression, $\mathrm{HC}$ : healthy controls. observed decreased $\mathrm{Kr}$ in the left caudate nucleus in $\mathrm{BD}$ compared with $\mathrm{HC}$ subjects and higher $\mathrm{Ka}$ in left putamen and lower MD in the right caudate nucleus in UD group than in $\mathrm{BD}$ group. In DKI, MK is a dimensionless parameter that reflects the average degree of diffusion restriction, while Ka and $\mathrm{Kr}$ measure the kurtoses along the directions parallel and perpendicular to the principal diffusion direction, respectively. ${ }^{43}$ Therefore, increased $\mathrm{Ka}$ and decreased $\mathrm{Kr}$ in caudate nucleus result in no significant changes of $\mathrm{MK}$ in this region. In gray matter, increased Ka may be due to broken axons, increase of basal dendrites, synaptic refinement, and cell density, which would cause more water diffusion restriction in the axial direction, leading to higher $\mathrm{Ka}^{32,44,45}$ in the left putamen and caudate nucleus in UD. The water diffusion is more restricted in UD in the left putamen due to the higher Ka in UD than in BD. These processes together with demyelination may result in more free water diffusion in the radial direction and hence lower $\mathrm{Kr}^{29,43}$ was observed in the left caudate nucleus both in $\mathrm{BD}$ and UD. The significant increase of $\mathrm{Ka}$ and decrease of $\mathrm{Kr}$ in the putamen and caudate nucleus is accompanied by no changes in Da and Dr which further suggests that kurtosis is more sensitive to the changes of microstructure integrity in gray matter, as also suggested by Jensen. ${ }^{26}$ Meanwhile, the MD value which may serve as a surrogate marker for cell and fiber density ${ }^{46}$ is lower in UD than in BD in the right caudate, it may result from subtle changes of cell density which didn't demonstrate in abnormality of parallel (Ka and $\mathrm{Da}$ ) and perpendicular ( $\mathrm{Kr}$ and $\mathrm{Dr}$ ) kurtosis and diffusion parameters. According to the above possible neuropathological changes, we speculate that these results may associate with volume reduction or atrophy. Results from literatures supported our speculation. Recent studies found both the BD and $\mathrm{UD}^{16}$ participants had smaller volumes for the caudate than

Table 5. ROC analyses of the significant parameters from MANOVA in UD and BD

\begin{tabular}{|c|c|c|c|c|c|}
\hline & AUC & $\mathrm{p}$ value & Cut-off value & Sensitivity (\%) & Specificity (\%) \\
\hline \multicolumn{6}{|c|}{ UD vs. HC } \\
\hline \multicolumn{6}{|c|}{ Left caudate nucleus head } \\
\hline $\mathrm{Ka}$ & 0.657 & 0.030 & 0.602 & 73.1 & 51.2 \\
\hline $\mathrm{Kr}$ & 0.726 & 0.002 & 0.554 & 84.6 & 60.5 \\
\hline $\mathrm{CBF}$ & 0.657 & 0.020 & 54.40 & 70.0 & 57.1 \\
\hline \multicolumn{6}{|c|}{ Left putamen } \\
\hline $\mathrm{Ka}$ & 0.685 & 0.013 & 0.718 & 70.8 & 56.1 \\
\hline $\mathrm{CBF}$ & 0.676 & 0.009 & 49.46 & 73.3 & 57.1 \\
\hline \multicolumn{6}{|l|}{ BD vs. HC } \\
\hline \multicolumn{6}{|c|}{ Left caudate nucleus head } \\
\hline $\mathrm{Kr}$ & 0.664 & 0.009 & 0.554 & 74.4 & 60.5 \\
\hline
\end{tabular}

ROC: receiver operating characteristic curves, MANOVA: multivariate analysis of variance, BD: bipolar disorder, UD: unipolar depression, HC: healthy controls, AUC: area under the curve, Ka: axial kurtosis, Kr: radial kurtosis, CBF: cerebral blood flow 
did the healthy participants and the reduced caudate volume was associated withthe depression severityin patients with UD. ${ }^{17}$ Another structural MR studies ${ }^{47}$ also found individuals with $\mathrm{BD}$ showed strong gray matter volume reductions in the caudate and putamen. ${ }^{48}$ Further, a resting-state functional MRI study ${ }^{49}$ found depressed patients had lower functional connectivity of the nucleus accumbens with the caudate and putamen. Similarly, Bluhm et al. ${ }^{50}$ also found deficits in default-mode network connectivity with the bilateral caudate in UD. Moreover, it's worth noting that among the five factors identified as significant parameters for UD from HC (Table 5), ROC analysis revealed the $\mathrm{Kr}$ in the left caudate nucleus head yield the highest predictive power for future diagnostic applications, and the Ka in the left putamen just followed by it. Taken together, the present microstructure changes in basal ganglia provide novel evidence for structural damage in UD and BD. The microstructure impairment in putamen and caudate nucleus may be an overlapping feature in the two disorders.

We also observed decreased CBF in patient with UD compared with $\mathrm{HC}$ in left putamen and caudate nucleus. An extensive literature review of positron emission tomography (PET) studies of depressed patients in the resting state revealed that patients with UD have reduced CBF and glucose metabolism in the caudate nucleus. ${ }^{51}$ A single photon emission computed tomography (SPECT) study by Fountoulakis et al. ${ }^{52}$ found global brain hypoperfusion including the basal ganglia in UD patients. Another SPECT study ${ }^{34}$ found severely depressed UD patients showed more cerebral perfusion deficits than less depressed patients in the basal ganglia, ${ }^{53}$ further support our findings. Unfortunately, we did not find any significant correlations between $\mathrm{CBF}$ and the clinical variables (HDRS scores, illness duration) in basal ganglia in patients with either BD or UD. However, these findings are somewhat contrary to a previous functional MRI study which showed hyperperfusion in the putamen in UD. ${ }^{35}$ Different reasons such as differences in sample size, subjects' ages, age of depression onset, medication status, response to treatment, comorbidities as well as differences in data acquisition and data preprocessing would lead to an inconsistent result could explain these conflicting findings. Besides, when compared with PET which currently regarded as the most accurate and precise method to measure CBF, ASL provides similar quantitative CBF as PET ${ }^{54,55}$ Furthermore, unlike PET techniques, ASL technique has the advantage of not using radioactive sources or injected contrast agents, showed a higher longitudinal repeatability for regional perfusion measurements, ${ }^{56}$ allowing for frequent scanning, especially useful in long term follow-up studies. Therefore, these findings confirmed that CBF abnormality existed in the basal ganglia in UD, suggest CBF changes in this region may be pathophysiologically important in depression.
Further studies which include large samples and homogeneous subjects are needed to confirm the CBF variations in the two disorders.

Prior neuroimaging studies have noted the importance of thalamus in BD and UD. ${ }^{57,58}$ Contrary to expectations, this study didn't show any significant difference in DKI parameters (microstructural integrity) and $\mathrm{CBF}$ in $\mathrm{BD}$ and UD group. In agreement with our findings, MacMaster et al. ${ }^{48}$ and Monkul et al. ${ }^{59}$ found no volumetric group differences between BD, UD and HC for the thalamus, which support our findings. However, several structural neuroimaging studies found volume reductions in the thalamus in $\mathrm{BD}^{60}$ and $\mathrm{UD}^{57,58} \mathrm{~A}$ metaanalysis also revealed volume reductions in the thalamus in late-life depression. ${ }^{61}$ Further, a resting-state functional MRI study by Alexopoulos et al. ${ }^{49}$ found depressed patients had lower functional connectivity of the nucleus accumbens with the thalamus. A monetary incentive delay task functional MRI study by Dichter et al. ${ }^{62}$ revealed the remitted UD group was characterized by relatively decreased activation in the left thalamus during the outcome phase of the task. Meanwhile, Lui et al. ${ }^{63}$ found the treatment-resistant depression patients showed disrupted functional connectivity mainly in thalamus areas bilaterally. Furthermore, in agreement with our results, a PET study ${ }^{64}$ revealed no significant changes in CBF in UD compared with $\mathrm{HC}$ in bilateral thalamus, nevertheless, the severity of depression was directly correlated with the blood flow in right thalamus. Besides, a SPECT study reported non-responders for repetitive transcranial magnetic stimulation of the pharmacoresistant depression patients showed CBF reduction in right thalamus. ${ }^{65}$ Taken together, the functional neuroimaging suggest that the thalamus plays a critical role in depression. However, it's still remains controversial when it comes to the structural (especially the volume variance) and perfusional changes in the thalamus. Our findings provide new evidences for the microstructural integrity and CBF changes of thalamus in BD and UD.

Several limitations to this pilot study need to be acknowledged. 1) The number of participants in the present study was not large, although larger than in many previous studies. It is possible that subtle differences between groups would have been detected in a larger sample size. 2) Parts of the patients included were under medication prior to MRI scanning. Furthermore, the medication varied, and the number of subjects was too limited to group them according to a specific type of drug. 3) The ROIs on the area of the caudate nucleus head, putamen and thalamus were drawn manually, and the reproducibility of measurements was unclear. However, all ROIs were drawn by two of the authors, rater bias was prevented by blinding, and the inter-class correlation coefficients were 0.704-0.983. 4) We did not use multiple comparison 
corrections when exploring the relationship between the clinical variables and DKI/3D ASL parameters. 5) Because DKI demands high b-value diffusion weighted images, it is sensitive to noise. Consequently, the resolution of the acquired images is suboptimal to sustain sufficiently high signal-to-noise ratios and to minimize acquisition time. This may have led to partial volume effects, which could have reduced the sensitivity of the analysis. 6) In 3D ASL, there only used one postlabelling delay time (1525 ms) in pCASL, which may have a potential impact on the evaluation of cerebral blood flow in some cases. ${ }^{66}$ 7) The current study is a cross-sectional study, although the present patients with UD had no family history of $\mathrm{BD}$, in the absence of longitudinal data we do not know whether some patients will later switch to BD. Thus, we will do the further longitudinal follow up investigation in the future study.

In conclusion, our findings are the first to reveal the microstructural integrity of basal ganglia and thalamus in the pathogenesis of BD and UD disorders in vivo. Our results suggest that these two disorders may have overlaps in microstructural abnormality in basal ganglia during the depressive period. The $\mathrm{Kr}$ value in the left caudate nucleus head may serve as a potential biomarker for UD. Decreased CBF in basal ganglia may be a key neurobiological feature of UD.

\section{Supplementary Materials}

The online-only Data Supplement is available with this article at https://doi.org/10.4306/pi.2017.14.4.471.

\section{Acknowledgments}

The study was supported by grants from the National Natural Science Foundation of China (81501456, 81471650); Natural Science Foundation of Guangdong Province, China (2014A030313375); Planned Science and Technology Project of Guangdong Province, China (2014B020212022); Planned Science and Technology Project of Guangzhou, China (1563000653); Fundamental Research Funds for the Central Universities, China (21615476).

\section{REFERENCES}

1. Judd LL, Akiskal HS, Schettler PJ, Endicott J, Maser J, Solomon DA, et al. The long-term natural history of the weekly symptomatic status of bipolar I disorder. Arch Gen Psychiatry 2002;59:530-537.

2. Hirschfeld RM, Lewis L, Vornik LA. Perceptions and impact of bipolar disorder: how far have we really come? Results of the national depressive and manic-depressive association 2000 survey of individuals with bipolar disorder. J Clin Psychiatry 2003;64:161-174.

3. Rubio JM, Markowitz JC, Alegria A, Perez-Fuentes G, Liu SM, Lin KH, et al. Epidemiology of chronic and nonchronic major depressive disorder: results from the national epidemiologic survey on alcohol and related conditions. Depress Anxiety 2011;28:622-631.

4. Kessler RC, Berglund P, Demler O, Jin R, Koretz D, Merikangas KR, et al. The epidemiology of major depressive disorder: results from the National Comorbidity Survey Replication (NCS-R). JAMA 2003;289: 3095-3105.

5. Kempton MJ, Salvador Z, Munafo MR, Geddes JR, Simmons A, Frangou $\mathrm{S}$, et al. Structural neuroimaging studies in major depressive disorder. Meta-analysis and comparison with bipolar disorder. Arch Gen
Psychiatry 2011;68:675-690.

6. Huyser C, Veltman DJ, de Haan E, Boer F. Paediatric obsessive-compulsive disorder, a neurodevelopmental disorder? Evidence from neuroimaging. Neurosci Biobehav Rev 2009;33:818-830.

7. Arsalidou M, Duerden EG, Taylor MJ. The centre of the brain: topographical model of motor, cognitive, affective, and somatosensory functions of the basal ganglia. Hum Brain Mapp 2013;34:3031-3054.

8. Garg A, Appel-Cresswell S, Popuri K, McKeown MJ, Beg MF. Morphological alterations in the caudate, putamen, pallidum, and thalamus in Parkinson's disease. Front Neurosci 2015;9:101.

9. Lai CH. Patterns of cortico-limbic activations during visual processing of sad faces in depression patients: a coordinate-based meta-analysis. J Neuropsychiatry Clin Neurosci 2014;26:34-43.

10. Womer FY, Wang L, Alpert KI, Smith MJ, Csernansky JG, Barch DM, et al. Basal ganglia and thalamic morphology in schizophrenia and bipolar disorder. Psychiatry Res 2014;223:75-83.

11. Xueying L, Zhongping Z, Zhoushe Z, Li G, Yongjin T, Changzheng S, et al. Investigation of apparent diffusion coefficient from ultra-high bvalues in parkinson's disease. Eur Radiol 2015;25:2593-2600.

12. He N, Ling H, Ding B, Huang J, Zhang Y, Zhang Z, et al. Region-specific disturbed iron distribution in early idiopathic Parkinson's disease measured by quantitative susceptibility mapping. Human Brain Mapp 2015;36:4407-4420.

13. Sanchez-Castaneda C, Cherubini A, Elifani F, Peran P, Orobello S, Capelli $\mathrm{G}$, et al. Seeking huntington disease biomarkers by multimodal, cross-sectional basal ganglia imaging. Human Brain Mapp 2013;34: 1625-1635.

14. Paulsen JS. Functional imaging in Huntington's disease. Exp Neurology 2009;216:272-277.

15. Shaw P, De Rossi P, Watson B, Wharton A, Greenstein D, Raznahan A, et al. Mapping the development of the basal ganglia in children with attention-deficit/hyperactivity disorder. J Am Acad Child Adolesc Psychiatry 2014;53:780-789.

16. Sacchet MD, Livermore EE, Iglesias JE, Glover GH, Gotlib IH. Subcortical volumes differentiate Major Depressive Disorder, Bipolar Disorder, and remitted Major Depressive Disorder. J Psychiatr Res 2015;68:91-98.

17. Pizzagalli DA, Holmes AJ, Dillon DG, Goetz EL, Birk JL, Bogdan R, et al. Reduced caudate and nucleus accumbens response to rewards in unmedicated individuals with major depressive disorder. Am J Psychiatry 2009;166:702-710.

18. Liberg B, Ekman CJ, Sellgren C, Johansson A, Landen M. Vertex-based morphometry in euthymic bipolar disorder implicates striatal regions involved in psychomotor function. Psychiatry Res 2014;221:173-178.

19. Serafini G, Pompili M, Borgwardt S, Houenou J, Geoffroy PA, Jardri R, et al. Brain changes in early-onset bipolar and unipolar depressive disorders: a systematic review in children and adolescents. Eur Child Adolesc Psychiatry 2014;23:1023-1041.

20. Womer FY, Wang L, Alpert KI, Smith MJ, Csernansky JG, Barch DM, et al. Basal ganglia and thalamic morphology in schizophrenia and bipolar disorder. Psychiatry Res 2014;223:75-83.

21. Fakhoury M. New insights into the neurobiological mechanisms of major depressive disorders. Gen Hosp Psychiatry 2015;37:172-177.

22. Lorenzetti V, Allen NB, Fornito A, Yucel M. Structural brain abnormalities in major depressive disorder: a selective review of recent MRI studies. J Affect Disord 2009;117:1-17.

23. Teng S, Lu CF, Wang PS, Hung CI, Li CT, Tu PC, et al. Classification of bipolar disorder using basal-ganglia-related functional connectivity in the resting state. Conf Proc IEEE Eng Med Biol Soc 2013;2013:10571060 .

24. Liu J, Ren L, Womer FY, Wang J, Fan G, Jiang W, et al. Alterations in amplitude of low frequency fluctuation in treatment-naive major depressive disorder measured with resting-state fMRI. Human Brain Mapp 2014;35:4979-4988.

25. Lu H, Jensen JH, Ramani A, Helpern JA. Three-dimensional characterization of non-gaussian water diffusion in humans using diffusion 
kurtosis imaging. NMR Biomed 2006;19:236-247.

26. Jensen JH, Helpern JA. MRI quantification of non-Gaussian water diffusion by kurtosis analysis. NMR Biomed 2010;23:698-710.

27. Gao Y, Zhang Y, Wong CS, Wu PM, Zhang Z, Gao J, et al. Diffusion abnormalities in temporal lobes of children with temporal lobe epilepsy: a preliminary diffusional kurtosis imaging study and comparison with diffusion tensor imaging. NMR Biomed 2012;25:1369-1377.

28. Jensen JH, Helpern JA, Ramani A, Lu H, Kaczynski K. Diffusional kurtosis imaging: the quantification of non-gaussian water diffusion by means of magnetic resonance imaging. Magn Reson Med 2005;53: 1432-1440.

29. Hui ES, Cheung MM, Qi L, Wu EX. Towards better MR characterization of neural tissues using directional diffusion kurtosis analysis. Neuroimage 2008;42:122-134.

30. Jensen JH, Falangola MF, Hu C, Tabesh A, Rapalino O, Lo C, et al. Preliminary observations of increased diffusional kurtosis in human brain following recent cerebral infarction. NMR Biomed 2011;24:452-457.

31. Kamagata K, Tomiyama H, Hatano T, Motoi Y, Abe O, Shimoji K, et al. A preliminary diffusional kurtosis imaging study of Parkinson disease: comparison with conventional diffusion tensor imaging. Neuroradiology 2014;56:251-258.

32. Helpern JA, Adisetiyo V, Falangola MF, Hu C, Di Martino A, Williams $\mathrm{K}$, et al. Preliminary evidence of altered gray and white matter microstructural development in the frontal lobe of adolescents with attentiondeficit hyperactivity disorder: a diffusional kurtosis imaging study. J Magn Reson Imaging 2011;33:17-23.

33. Zhu J, Zhuo C, Qin W, Wang D, Ma X, Zhou Y, et al. Performances of diffusion kurtosis imaging and diffusion tensor imaging in detecting white matter abnormality in schizophrenia. Neuroimage Clin 2015;7: 170-176.

34. Vardi N, Freedman N, Lester H, Gomori JM, Chisin R, Lerer B, et al. Hyperintensities on T2-weighted images in the basal ganglia of patients with major depression: cerebral perfusion and clinical implications. Psychiatry Res 2011;192:125-130.

35. Ho TC, Wu J, Shin DD, Liu TT, Tapert SF, Yang G, et al. Altered cerebral perfusion in executive, affective, and motor networks during adolescent depression. J Am Acad Child Adolesc Psychiatry 2013;52:1076-1091.

36. Zhao L, Wang Y, Jia Y, Zhong S, Sun Y, Zhou Z, et al. Cerebellar microstructural abnormalities in bipolar depression and unipolar depression: a diffusion kurtosis and perfusion imaging study. J Affect Disord 2016;195:21-31.

37. Walther S, Hofle O, Federspiel A, Horn H, Hugli S, Wiest R, et al. Neural correlates of disbalanced motor control in major depression. J Affect Disord 2012;136:124-133.

38. Ho TC, Wu J, Shin DD, Liu TT, Tapert SF, Yang G, et al. Altered cerebral perfusion in executive, affective, and motor networks during adolescent depression. J Am Acad Child Adolesc Psychiatry 2013;52:1076-1091.

39. Alsop DC, Detre JA. Reduced transit-time sensitivity in noninvasive magnetic resonance imaging of human cerebral blood flow. J Cereb Blood Flow Metab 1996;16:1236-1249.

40. Wang J, Zhang Y, Wolf RL, Roc AC, Alsop DC, Detre JA. Amplitudemodulated continuous arterial spin-labeling 3.0-T perfusion MR imaging with a single coil: feasibility study. Radiology 2005;235:218-228.

41. Jarnum H, Steffensen EG, Knutsson L, Frund ET, Simonsen CW, Lundbye-Christensen S, et al. Perfusion MRI of brain tumours: a comparative study of pseudo-continuous arterial spin labelling and dynamic susceptibility contrast imaging. Neuroradiology 2010;52:307-317.

42. Wu B, Lou X, Wu X, Ma L. Intra- and interscanner reliability and reproducibility of $3 \mathrm{D}$ whole-brain pseudo-continuous arterial spin-labeling MR perfusion at 3T. J Magn Reson Imaging 2014;39:402-409.

43. Sun Y, Sun J, Zhou Y, Ding W, Chen X, Zhuang Z, et al. Assessment of in vivo microstructure alterations in gray matter using DKI in internet gaming addiction. Behav Brain Funct 2014;10:37.

44. Zhuo J, Xu S, Proctor JL, Mullins RJ, Simon JZ, Fiskum G, et al. Diffusion kurtosis as an in vivo imaging marker for reactive astrogliosis in traumatic brain injury. Neuroimage 2012;59:467-477.

45. Gao J, Feng ST, Wu B, Gong N, Lu M, Wu PM, et al. Microstructural brain abnormalities of children of idiopathic generalized epilepsy with generalized tonic-clonic seizure: a voxel-based diffusional kurtosis imaging study. J Magn Reson Imaging 2015;41:1088-1095.

46. Abe O, Yamasue H, Kasai K, Yamada H, Aoki S, Inoue H, et al. Voxelbased analyses of gray/white matter volume and diffusion tensor data in major depression. Psychiatry Res 2010;181:64-70.

47. Redlich R, Almeida JJ, Grotegerd D, Opel N, Kugel H, Heindel W, et al. Brain morphometric biomarkers distinguishing unipolar and bipolar depression. A voxel-based morphometry-pattern classification approach. JAMA Psychiatry 2014;71:1222-1230.

48. MacMaster FP, Carrey N, Langevin LM, Jaworska N, Crawford S. Disorder-specific volumetric brain difference in adolescent major depressive disorder and bipolar depression. Brain Imaging Behav 2014;8:119127.

49. Alexopoulos GS, Hoptman MJ, Yuen G, Kanellopoulos D, Seirup JK, Lim KO, et al. Functional connectivity in apathy of late-life depression: A preliminary study. J Affect Disord 2013;149:398-405.

50. Bluhm R, Williamson P, Lanius R, Theberge J, Densmore M, Bartha R, et al. Resting state default-mode network connectivity in early depression using a seed region-of-interest analysis: decreased connectivity with caudate nucleus. Psychiatry Clin Neurosci 2009;63:754-761.

51. Videbech P. PET measurements of brain glucose metabolism and blood flow in major depressive disorder: a critical review. Acta Psychiatr Scand 2000;101:11-20.

52. Fountoulakis KN, Iacovides A, Gerasimou G, Fotiou F, Ioannidou C, Bascialla $\mathrm{F}$, et al. The relationship of regional cerebral blood flow with subtypes of major depression. Prog Neuropsychopharmacol Biol Psychiatry 2004;28:537-546.

53. Ebmeier KP, Prentice N, Ryman A, Halloran E, Rimmington JE, Best $\mathrm{JK}$, et al. Temporal lobe abnormalities in dementia and depression: a study using high resolution single photon emission tomography and magnetic resonance imaging. J Neurol Neurosurg Psychiatry 1997; 63:597-604.

54. Heijtel DFR, Mutsaerts H, Bakker E, Schober P, Stevens MF, Petersen ET, et al. Accuracy and precision of pseudo-continuous arterial spin labeling perfusion during baseline and hypercapnia: a head-to-head comparison with $15 \mathrm{O} \mathrm{H2O}$ positron emission tomography. Neuroimage 2014;92:182-192.

55. Schmid S, Heijtel DF, Mutsaerts HJ, Boellaard R, Lammertsma AA, Nederveen AJ, et al. Comparison of velocity- and acceleration-selective arterial spin labeling with [15O]H2O positron emission tomography. J Cereb Blood Flow Metab 2015;35:1296-1303.

56. Kilroy E, Apostolova L, Liu C, Yan L, Ringman J, Wang DJ. Reliability of two-dimensional and three-dimensional pseudo-continuous arterial spin labeling perfusion MRI in elderly populations: comparison with 15O-water positron emission tomography. J Magn Reson Imaging 2014; 39:931-939.

57. Vasic N, Walter H, Hoese A, Wolf RC. Gray matter reduction associated with psychopathology and cognitive dysfunction in unipolar depression: a voxel-based morphometry study. J Affect Disord 2008;109:107-116.

58. Nugent AC, Davis RM, Zarate CA Jr, Drevets WC. Reduced thalamic volumes in major depressive disorder. Psychiatry Res 2013;213:179-185.

59. Monkul ES, Nicoletti MA, Spence D, Sassi RB, Axelson D, Brambilla P, et al. MRI study of thalamus volumes in juvenile patients with bipolar disorder. Depress Anxiety 2006;23:347-352.

60. Rimol LM, Hartberg CB, Nesvag R, Fennema-Notestine C, Hagler DJ, Jr, Pung CJ, et al. Cortical thickness and subcortical volumes in schizophrenia and bipolar disorder. Biol Psychiatry 2010;68:41-50.

61. Sexton CE, Mackay CE, Ebmeier KP. A systematic review and metaanalysis of magnetic resonance imaging studies in late-life depression. Am J Geriatr Psychiatry 2013;21:184-195.

62. Dichter GS, Kozink RV, McClernon FJ, Smoski MJ. Remitted major depression is characterized by reward network hyperactivation during 
reward anticipation and hypoactivation during reward outcomes. J Affect Disord 2012;136:1126-1134.

63. Lui S, Wu Q, Qiu L, Yang X, Kuang W, Chan RC, et al. Resting-state functional connectivity in treatment-resistant depression. Am J Psychiatry 2011;168:642-648.

64. Monkul ES, Silva LA, Narayana S, Peluso MA, Zamarripa F, Nery FG, et al. Abnormal resting state corticolimbic blood flow in depressed unmedicated patients with major depression: a (15)O-H(2)O PET study. Human Brain Mapp 2012;33:272-279.
65. Richieri R, Boyer L, Farisse J, Colavolpe C, Mundler O, Lancon C, et al. Predictive value of brain perfusion SPECT for rTMS response in pharmacoresistant depression. Eur J Nucl Med Mol Imaging 2011;38: 1715-1722.

66. Xiao HF, Chen ZY, Lou X, Wang YL, Gui QP, Wang Y, et al. Astrocytic tumour grading: a comparative study of three-dimensional pseudocontinuous arterial spin labelling, dynamic susceptibility contrast-enhanced perfusion-weighted imaging, and diffusion-weighted imaging. Eur Radiol 2015;25:3423-3430. 